\title{
Noninvasive Evaluation of CBF and Perfusion Delay of Moyamoya Disease Using Arterial Spin-Labeling MRI with Multiple Postlabeling Delays: Comparison with ${ }^{15}$ O-Gas PET and DSC-MRI
}

\author{
(D) S. Hara, (D). Tanaka, (D)Y. Ueda, (D). Hayashi, (D). Inaji, (D). Ishiwata, (D). Ishii, (D)T. Maehara, and (D). Nariai \\ O- $\equiv$
}

\begin{abstract}
BACKGROUND AND PURPOSE: Arterial spin-labeling MR imaging with multiple postlabeling delays has a potential to evaluate various hemodynamic parameters. To clarify whether arterial spin-labeling MR imaging can identify CBF and perfusion delay in patients with Moyamoya disease, we compared arterial spin-labeling, DSC, and ${ }^{15} \mathrm{O}$-gas PET in terms of their ability to identify these parameters.
\end{abstract}

MATERIALS AND METHODS: Eighteen patients with Moyamoya disease (5 men, 13 women; ages, 21-55 years) were retrospectively analyzed. CBF values of pulsed continuous arterial spin-labeling using 2 postlabeling delays (short arterial spin-labeling, 1525 ms; delayed arterial spin-labeling, $2525 \mathrm{~ms}$ ) were compared with CBF values measured by ${ }^{15} \mathrm{O}$-gas PET. All plots were divided into 2 groups by the cutoff of time-based parameters (the time of the maximum observed concentration, TTP, MTT, delay of MTT to cerebellum, and disease severity [symptomatic or not]). The ratio of 2 arterial spin-labeling CBFs (delayed arterial spin-labeling CBF to short arterial spin-labeling CBF) was compared with time-based parameters: time of the maximum observed concentration, TTP, and MTT.

RESULTS: The short arterial spin-labeling-CBF values were significantly correlated with the PET-CBF values $(r=0.63 ; P=.01)$. However, the short arterial spin-labeling-CBF value dropped in the regions with severe perfusion delay. The delayed arterial spin-labeling CBF overestimated PET-CBF regardless of the degree of perfusion delay. Delayed arterial spin-labeling CBF/short arterial spin-labeling CBF was well correlated with the time of the maximum observed concentration, TTP, and MTT ( $\rho=0.71,0.64$, and 0.47 , respectively).

CONCLUSIONS: Arterial spin-labeling using 2 postlabeling delays may detect PET-measured true CBF and perfusion delay in patients with Moyamoya disease. Provided its theoretic basis and limitations are considered, noninvasive arterial spin-labeling could be a useful alternative for evaluating the hemodynamics of Moyamoya disease.

ABBREVIATIONS: ASL = arterial spin-labeling; dASL = delayed arterial spin-labeling; MTT delay = delay of MTT to cerebellum; PLD = postlabeling delay; sASL = short arterial spin-labeling; Tmax $=$ time of the maximum observed concentration

M oyamoya disease is a slowly progressive cerebrovascular disease with occlusion of the terminal portion of the internal carotid arteries. ${ }^{1-3}$ Perfusion studies are indispensable

Received August 15, 2016; accepted after revision November 9.

From the Department of Neurosurgery (S.H., Y.T., Y.U., S.H., M.I., T.M., T.N.), Tokyo Medical and Dental University, Tokyo, Japan; and Research Team for Neuroimaging (M.I., K. Ishiwata, K. Ishii, T.N.), Tokyo Metropolitan Institute of Gerontology, Tokyo, Japan.

This research was funded in part by a Japan Society for the Promotion of Science KAKENHI grant (15K10294) and by a SENSHIN Medical Research Foundation grant. The authors have no personal financial or institutional interest in any of the drugs, materials, or devices described in this article.

Please address correspondence to Yoji Tanaka, MD, PhD, Department of Neurosurgery, Tokyo Medical and Dental University, 1-5-45 Yushima, Bunkyo-ku, Tokyo 113-8519, Japan; e-mail: tanaka.nsrg@tmd.ac.jp

- Indicates open access to non-subscribers at www.ajnr.org

$\equiv$ Indicates article with supplemental on-line tables.

Indicates article with supplemental on-line photos.

http://dx.doi.org/10.3174/ajnr.A5068 for determining the most appropriate treatment strategy for individual patients with this disease because the hemodynamic conditions are highly variable among patients. ${ }^{4}$ Another characteristic of Moyamoya disease is its prevalence among children and adolescents, which underscores the need for truly noninvasive studies.

To understand the hemodynamic status of patients with Moyamoya disease, it is important to evaluate CBF and various time-based parameters. ${ }^{15} \mathrm{O}$-gas $\mathrm{PET}$ provides quantitative $\mathrm{CBF}$ values by using a diffusible tracer and calculations by the KetySchmidt equation, and thus is considered a criterion standard technique. However, the procedure is costly and not readily available at most institutes, and the radiation exposure makes it difficult to apply this technique repeatedly for young or juvenile patients. In contrast, DSC is free of ionized radiation, easily available at most institutes, and can calculate time-based parameters such as the time of the maximum observed concentration (Tmax), TTP, and MTT, which have been reported to be important clinical 
biomarkers in Moyamoya disease. ${ }^{5-7}$ Nevertheless, DSC still requires an injection of contrast media.

Arterial spin-labeling MR imaging (ASL) has emerged as a noninvasive technique for evaluating cerebral hemodynamics ${ }^{8-11}$ because it uses magnetically labeled water as an endogenous tracer. For the quantitation of CBF, ASL uses a mechanism similar to that of PET. The main problem with the ASL technique is its inferior SNR, but the recent spread of high-magnetic-field clinical MR imaging systems has made this method applicable in many clinical centers, providing high-quality CBF images. ASL has been applied to various fields, ${ }^{12-14}$ and several studies have reported a correlation between ASL-CBF and PET-CBF in healthy subjects, patients with Alzheimer disease, patients with occlusive atherosclerotic cerebrovascular disease, and children with Moyamoya disease. ${ }^{15-20}$ ASL with multiple postlabeling delays (PLDs) may also be used to evaluate time-based parameters and angiographic collateral flows, as suggested in some studies of symptomatic atherosclerotic cerebrovascular disease. ${ }^{21-23}$

We considered that noninvasive ASL could be appropriate for the clinical management of Moyamoya disease and that ASL might detect $\mathrm{CBF}$ and perfusion delay of the patients. Numerous ASL studies have been conducted in patients with Moyamoya disease, ${ }^{20,24-31}$ but studies simultaneously comparing ASL with both ${ }^{15} \mathrm{O}$-gas PET and time-based parameters of DSC are quite rare. In the present study, to verify the proposed usefulness of ASL, we compared ASL-CBF values obtained when using 2 PLDs with the data obtained by DSC and ${ }^{15} \mathrm{O}$-gas PET in patients with Moyamoya disease.

\section{MATERIALS AND METHODS Patients and Management Protocol}

The protocol of this retrospective study was approved by the ethics committee of Tokyo Medical and Dental University, where this study was conducted. We reviewed medical records from April 2011 to March 2013 and found 25 patients with Moyamoya disease who were evaluated with ASL, DSC, and ${ }^{15} \mathrm{O}$-gas PET. All patients were diagnosed as having Moyamoya disease by MR imaging and/or cerebral angiography according to the diagnostic guidelines. ${ }^{3}$ Seven patients were excluded from analysis: 1 patient with hemorrhagic onset, 1 patient who developed new infarction between MR imaging and PET examinations, and 5 others with long MR imaging-PET interval ( $>45$ days). The remaining 18 patients were 5 men and 13 women, ranging in age from $21-55$ years (mean age, 36 years). Nine of 36 hemispheres were considered to be symptomatic, 8 were postoperative (indirect bypass surgery $>1$ year before enrollment in this analysis), and the others were regarded as asymptomatic. The interval between MR imaging (ASL, DSC, and conventional MR imaging) and PET was 6-41 days (mean, 23.7 days). No intervention was performed, and no new symptoms developed over this interval. Patients were not controlled for caffeine intake or other activities before the imaging studies. The details of the patient characteristics, including MRA scores and stages, ${ }^{3,32}$ are listed in On-line Table 1.

\section{MR Imaging Perfusion Protocols}

We performed all MR imaging studies at our hospital with a $3 \mathrm{~T}$ MR scanner (Signa HDxt; GE Healthcare, Milwaukee, Wiscon- sin) with an 8-channel head coil. In addition to routine imaging sequences, including MRA and FLAIR, we acquired 3D pulsed continuous ASL images using the following parameters: TR, 4521 ms; TE, $9.8 \mathrm{~ms}$; FOV, $24 \mathrm{~cm}$; 512 sampling points on 8 spirals (matrix size of $512 \times 8$ ); spatial resolution, $5.0 \mathrm{~mm}$; section thickness, $4 \mathrm{~mm}$; number of sections, 30; excitations, 3; bandwidth, $62.50 \mathrm{~Hz}$; and labeling time, 1.5 seconds. We used 2 PLDs, 1525 $\mathrm{ms}$ and $2525 \mathrm{~ms}$, which we designated the short ASL (sASL) and delayed ASL (dASL), respectively.

We adopted the sequence of DSC used in a previous study: ${ }^{5}$ gradient recalled-echo single-shot multisection EPI with TR, 1000 $\mathrm{ms}$; TE, $40 \mathrm{~ms}$; FOV, $22 \mathrm{~cm}$; and matrix, $128 \times 128$. A series of 7.5 $\mathrm{mm}$-thick sections separated by $7.5-\mathrm{mm}$ gaps was acquired after a bolus injection of gadodiamide $(0.2 \mathrm{mmol} / \mathrm{kg}$ body weight; Omniscan 32\% [GE Healthcare]) via an antecubital vein by using a power injector (Nemoto Kyorindo, Tokyo, Japan) at the rate of $3 \mathrm{~mL} / \mathrm{s}$, followed by a $15-\mathrm{mL}$ saline flush.

\section{MR Imaging CBF Analysis}

The quantitative ASL-CBF map was generated on an Advantage Windows workstation (GE Healthcare) by using Functool software provided by the scanner manufacturer based on a theory described elsewhere. ${ }^{15}$

The DSC-MR imaging data were transferred to a personal computer and analyzed with the commercial software package Dr. View R2.5 (AJS, Tokyo, Japan). In preparation, we calculated the transverse relaxation rate $\left(\Delta \mathrm{R} 2^{*}\right)$ by the equation $\Delta \mathrm{R} 2^{*}(\mathrm{t})=$ $-\ln [\mathrm{S}(\mathrm{t}) / \mathrm{S} 0] / \mathrm{TE}$, where $\mathrm{S}(\mathrm{t})$ is the signal intensity at time $t$ and $\mathrm{S} 0$ is the precontrast baseline signal intensity. We generated a map of Tmax values by deconvolving the change in tissue concentration over the first pass of the contrast agent with an arterial input function by using singular value decomposition. ${ }^{33,34}$ The arterial input function was automatically obtained from ROIs that were manually placed on the internal carotid artery in each patient. A relative TTP map and a relative MTT map were generated directly from the time- $\Delta \mathrm{R} 2^{\star}$ curve.

\section{PET Protocol}

The PET study was carried out by using a SET 2400W scanner (Shimadzu, Kyoto, Japan) in the PET center at the Tokyo Metropolitan Institute of Gerontology. CBF was measured by continuous and consecutive 9-minute inhalations of $\mathrm{C}^{15} \mathrm{O}_{2}$ with continuous arterial blood sampling, using a table-lookup technique. ${ }^{35,36}$ The regional $\mathrm{CBF}$ value was obtained by calculating the values with lookup tables created from the arterial whole blood and plasma radioactivity curves and then correcting them for delay and dispersion. ${ }^{37}$

\section{Analysis of ROIs}

PET and MR images were spatially coregistered by using the image registration function of Dr. View R2.5. 3D data of PET-CBF and ASL-CBF were reconstructed into 7 sections matched to DSC. The ROIs were manually drawn on the raw images of DSC over 10 cortical areas (the bilateral frontal, parietal, rolandic, temporal, and occipital lobes) and the cerebellum in reference to the previous studies, ${ }^{5,16}$ as shown in Fig 1 and On-line Fig 1. Eight areas of 4 patients containing cortical infarction (see On-line Table 1 for details) were excluded from analysis. 


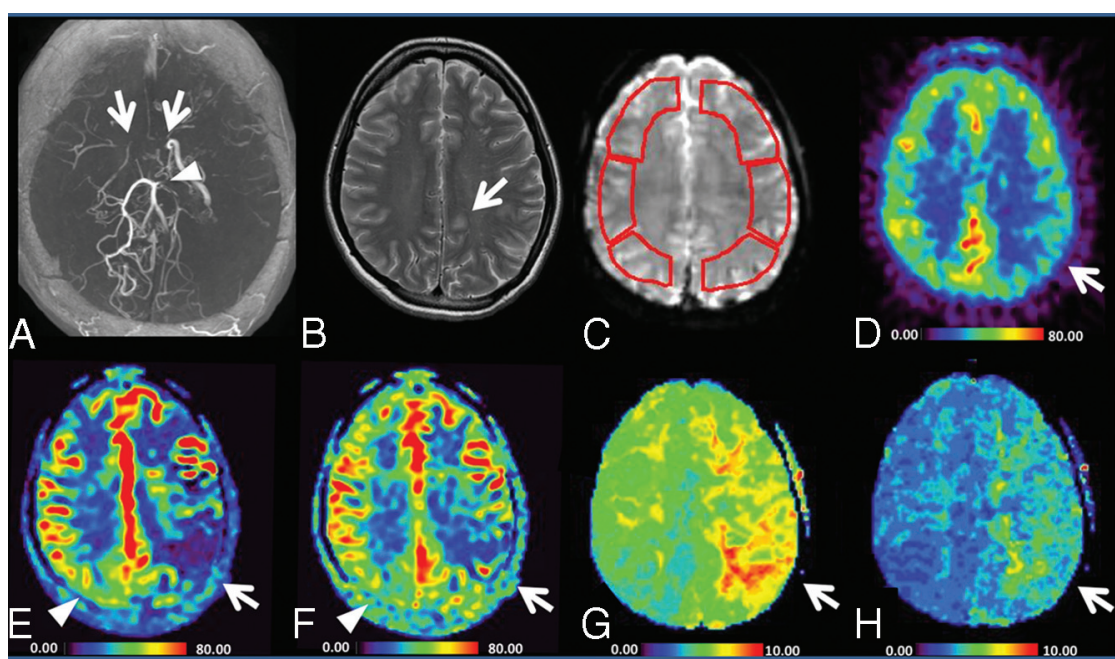

FIG 1. Images of a 21-year-old woman (patient 6 in On-line Table 1) with transient weakness of the right hand. The MRA ( $A$ ) shows occlusion of the bilateral terminal ICA (arrow), absence of the bilateral MCA, and stenosis of the left posterior cerebral artery (arrowhead). T2WI reveals a small ischemic change of the left hemisphere $(B)$. C, Sample ROIs used for the quantification of parameters. Color maps of PET-CBF, sASL-CBF, dASL-CBF, DSC-Tmax, and DSC-TTP values are shown from $D-H$, respectively. The ASL-CBF maps $(E, F)$ were visually comparable with the PET-CBF map $(D)$, but showed generally higher values than those of PET. The SASL-CBF $(E)$ shows lower values than dASL-CBF (F) on the affected side (arrows). Conversely, SASL-CBF shows higher values than dASL-CBF (arrowheads) on the less-affected side.

\section{Statistical Analysis}

All statistical analysis was performed with the commercial software package JMP version 11 (SAS Institute, Cary, North Carolina). The distribution of each value was evaluated with the Shapiro-Wilk test for normality, and values of $P<.05$ were regarded to be significant.

The ASL-CBF and DSC-CBF values were compared with PETCBF in all regions by using Bland-Altman plots, and Pearson correlation coefficients $(r)$ were calculated and tested for significance. The difference of $r$ values between the 2 groups was assessed by Fisher $z$ transformation, and values of $z>1.96$ were regarded to be significant. Next, to understand how the transit delay affected ASL-CBF values, all ROIs were divided into 2 groups by the following cutoffs: Tmax, 6.0 seconds; TTP, 4.0 seconds; MTT, 4.0 seconds; and delay of MTT to cerebellum (MTT delay), 1.5 and 2.0 seconds. These MTT delay values were previously shown to divide elevated CBV and oxygen extraction fraction. ${ }^{5}$ All plots were also divided into those with asymptomatic/ postoperative hemispheres and those with symptomatic sides to assess the effect of disease severity on ASL-CBF measurement. A paired $t$ test was performed to reveal differences between the 2 groups, and values of $P<.05$ were regarded to be significant.

Finally, the ratio of 2 ASLs (dASL-CBF to sASL-CBF) was compared with Tmax, TTP, and MTT to evaluate whether ASL with 2 PLDs can provide accurate information on the perfusion delay. Spearman correlation coefficients $(\rho)$ were calculated and tested for significance.

\section{RESULTS}

A representative case is shown in Fig 1. The ASL-CBF maps were visually comparable with the PET-CBF maps, but showed generally higher values than those of PET. The areas with low ASL-CBF values and the areas with a perfusion delay determined with
DSC parameters corresponded well. The ASL-CBF maps of the 2 PLDs were visually different. Short ASL-CBF showed lower values than dASL-CBF in the more hemodynamically impaired hemisphere with a longer delay in perfusion time (indicated by the arrows in Fig 1). Conversely, sASL-CBF showed higher values than dASL-CBF (the arrowheads in Fig 1) in the less-affected hemisphere.

\section{Correlation between ASL-CBF and PET-CBF}

The summary of CBF values of ASL and PET is shown in On-line Table 2. The CBF values of ASL and PET were normally distributed $(P=.08$ for sASL$\mathrm{CBF} ; P=.27$ for dASL-CBF; and $P=.32$ for PET-CBF). The sASL-CBF values were better correlated with PET-CBF $(r=0.63 ; P=.01[$ Fig 2A1]) compared with the dASL-CBF $(r=0.39 ; P<.0001$ in Fig 2B1) and DSC-CBF values $(r=$ $-0.15 ; P=.046$ [On-line Fig 2]) with statistical significance $(z=2.90$ for sASL-CBF versus dASL-CBF and $z=10.30$ for sASL-CBF versus DSC-CBF). The value of sASL-CBF minus PET-CBF was $1.72 \mathrm{~mL} / \mathrm{min} / 100 \mathrm{~g}$ (95\% CI, 0.50-2.94; dotted lines in Fig 2A2). When we divided all regions into 2 groups by the degree of perfusion delay and the disease severity, a significant correlation was seen between sASL-CBF and PET-CBF in the areas with smaller values of time-based parameters and in the less-affected hemispheres $(r=0.61 ; P<.0001$ for Tmax $\leq 6.0$ seconds [Fig 3A1]; $r=0.58, P=.001$ for TTP $\leq 4.0$ seconds; $r=0.54, P=.001$ for MTT $\leq 4.0$ seconds; $r=0.57, P=$ .001 for MTT delay $\leq 1.5$ seconds [On-line Fig 3A1]; and $r=0.63$, $P=.03$ in asymptomatic/postoperative hemispheres [On-line Fig $4 A 1])$. The difference between sASL-CBF and PET-CBF was significantly smaller in regions with long Tmax and TTP than in the others $(2.82 \mathrm{~mL} / \mathrm{min} / 100 \mathrm{~g}$ in Tmax $\leq 6.0$ seconds versus -2.02 in Tmax $>6.0$ seconds, $P=.002$ [Fig 3A2, B2] ; and 2.21 in TTP $\leq 4.0$ seconds versus -4.31 in TTP $>4.0$ seconds, $P=.01$ [On-line Table 3]). Taken together, these results showed that the sASL-CBF value dropped in the regions with severe perfusion delay.

On the other hand, the correlation between dASL-CBF and PET-CBF was moderate $(r=0.39, P<.0001$ [Fig 2B1]), and the difference between dASL-CBF and PET-CBF was $6.39 \mathrm{~mL} / \mathrm{min} /$ $100 \mathrm{~g}$ (95\% CI, 5.10-7.67 [Fig 2B2]), which was higher than the gap between sASL-CBF and PET-CBF $(P<.0001)$. The CBF values of dASL were higher than those of PET regardless of the degree of perfusion delay and the disease severity. The correlation of $\mathrm{dASL}-\mathrm{CBF}$ and PET-CBF was better in areas with delayed perfusion $(r=0.56,0.82,0.70$, and 0.65 in areas with Tmax $>6.0$ seconds [Fig 3D1], TTP $>4.0$ seconds, MTT $>4.0$ seconds, and MTT delay $>1.5$ seconds, respectively; $P=.002$ for TTP $>4.0$ seconds and $P<.0001$ for all other groups) than regions with less perfusion delay. The difference between dASL-CBF and PET-CBF 


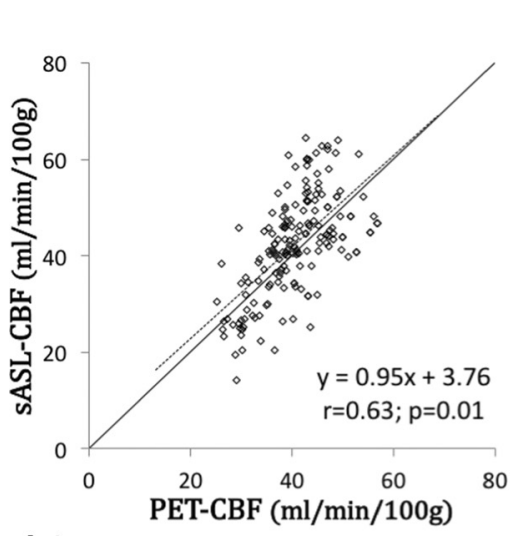

A1

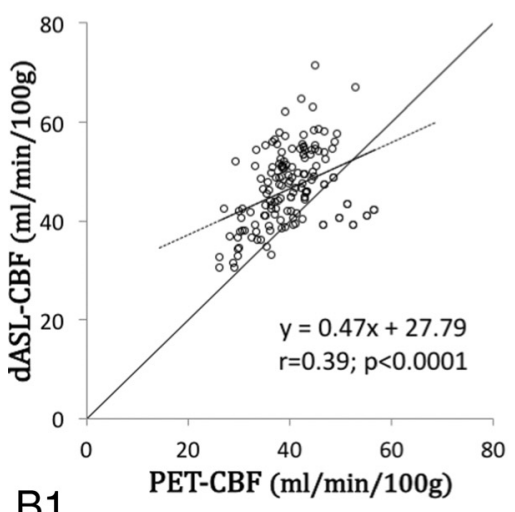

B1
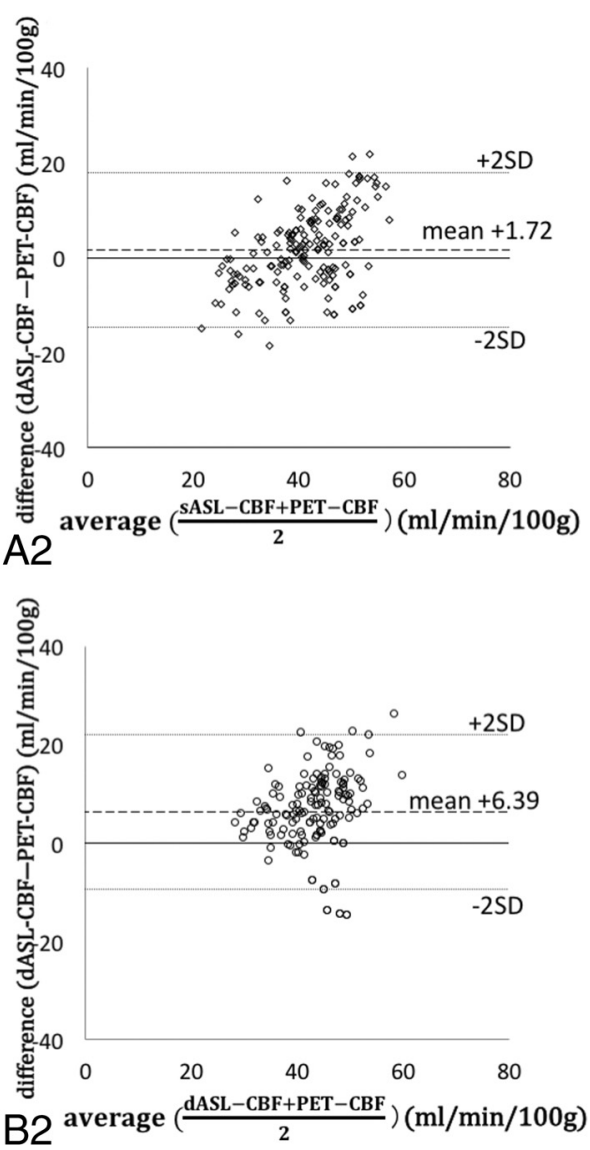

FIG 2. The relationship between regional $C B F$ values of $P E T$ and $A S L$. $A$ virtual straight line of $P E T-C B F$ equal to $S A S L-C B F$ is drawn in $A 7$ and $B 1$. The $S A S L-C B F$ grossly reflects the true CBF, whereas dASL-CBF grossly overestimates the true CBF.

was significantly higher in areas with long Tmax, MTT, and MTT delay than in areas with short time-based parameters (5.48 in Tmax $\leq 6.0$ seconds versus 9.16 in Tmax $>6.0$ seconds, $P=.01$ [Fig 3C2, D2]; 5.56 in MTT $\leq 4.0$ seconds versus 8.12 in MTT $>4.0$ seconds, $P=.04 ; 5.77$ in MTT delay $\leq 1.5$ seconds versus 10.04 in MTT delay $>1.5$ seconds, $P=.02$ ). Please see On-line Table 4 for details of the results.

\section{Correlations among dASL-CBF to SASL-CBF Ratio and Tmax, MTT, and TTP}

Because the ratio of dASL-CBF to sASL-CBF values was not normally distributed $(P<.0001)$, Spearman correlation coefficients ( $\rho$ ) were calculated between the ratio of dASL-CBF to SASL-CBF and DSC parameters. As shown in Fig 4, the ratio of dASL-CBF to sASL-CBF was well correlated with all 3 DSC parameters, Tmax, TTP, and MTT ( $\rho=0.71,0.64$, and 0.47 , respectively; $P<.0001$ for all).

\section{DISCUSSION}

Our findings demonstrated that sASL-CBF grossly reflected true $\mathrm{CBF}$ as measured by the criterion standard method PET and that CBF measurement by ASL was influenced by the regional delay of perfusion time and the disease severity. Moreover, our study suggested that ASL measurement with 2 PLDs could be used to evaluate the severity of perfusion delay.

Although ASL uses protons in blood as a diffusible tracer and

has an underlying mechanism similar to that of PET, it is also affected by the arterial transit time. Although many studies have reported a good correlation between the CBF values of ASL and PET, ${ }^{15-20}$ Ye et al $^{15}$ reported an underestimation of white matter CBF by ASL, which may have been related to the longer arterial transit times of the white matter regions compared with gray matter regions. Kimura et $\mathrm{al}^{16}$ revealed that the arterial transit time affected the perfusion signal of ASL and noted that a long transit time may lead to underestimation of the affected side in occlusive cerebrovascular disease. In our study, the CBF values of ASL essentially overestimated the PET-measured true CBF of Moyamoya disease and only in areas with long transit time when using sASL turned toward underestimation of true $\mathrm{CBF}$. We also found that sASL-CBF showed a better correlation with PETCBF than dASL-CBF. This overestimation was possibly related to our pulsed continuous ASL method itself. For example, the ASL-CBF values of normal healthy volunteers in our institute obtained by using the same cortical ROI analysis were $64.4 \mathrm{~mL} / \mathrm{min} / 100 \mathrm{~g}$ on average for sASL and $60.4 \mathrm{~mL} / \mathrm{min} / 100 \mathrm{~g}$ for dASL, whereas the ${ }^{15} \mathrm{O}$-gas PETmeasured CBFs of healthy subjects were reported to be 40.7-42.8 $\mathrm{mL} / \mathrm{min} / 100 \mathrm{~g} .{ }^{4}$ The difference between ASL-CBF and PET-CBF in normal healthy adults was approximately $20 \mathrm{~mL} / \mathrm{min} / 100 \mathrm{~g}$ in our institute. In our patients with Moyamoya disease, almost all the brain regions had some degree of perfusion delay, and ASL$\mathrm{CBF}$ in these patients was lower than that in normal healthy volunteers, but still higher than PET-CBF. Thus, the good correlation between the CBF values of SASL and PET was attributed to this offset effect of long transit time when PLD was short. In addition, the better SNR in short PLD would have contributed to the good correlation. In theory, the ASL signal is small and requires better SNR for accurate CBF measurement. Alsop et $\mathrm{al}^{14}$ pointed out that there is a fundamental trade-off in this relation: a short delay does not allow for complete delivery of the labeled blood water to the tissue, whereas a long delay results in strong T1 decay and, therefore, reduced SNR. In Moyamoya disease, the degree of transit delay varies even within individual regions of a single patient. Therefore, it is possible both that short PLD may increase the accuracy of CBF measurement because of the improved SNR and that long PLD may increase the accuracy of CBF measurement by allowing for greater delivery of labeled water to the tissue in patients with Moyamoya disease. We speculated that the former effect by short PLD might overcome the latter effect by long $\mathrm{PLD}$, resulting in a greater increase in the correlation between sASL-CBF and PET-CBF. 
$\operatorname{Tmax} \leq 6.0 \mathrm{~s}$

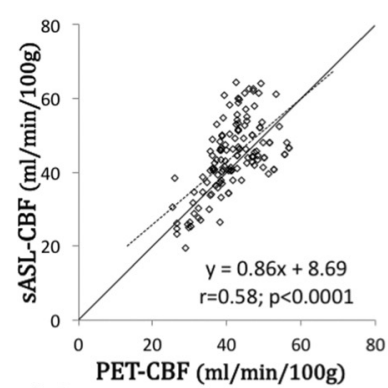

A1

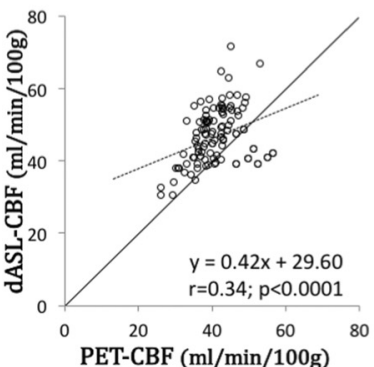

C1

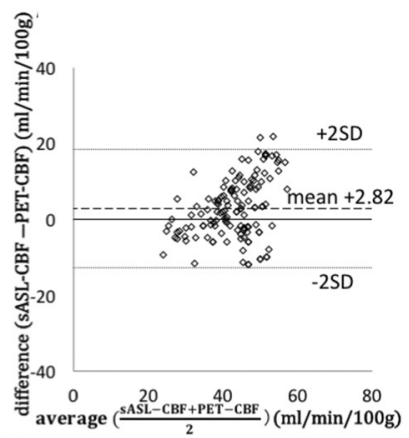

A2

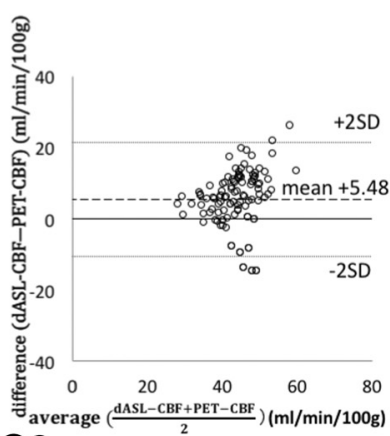

C2
Tmax $>6.0 \mathrm{~s}$

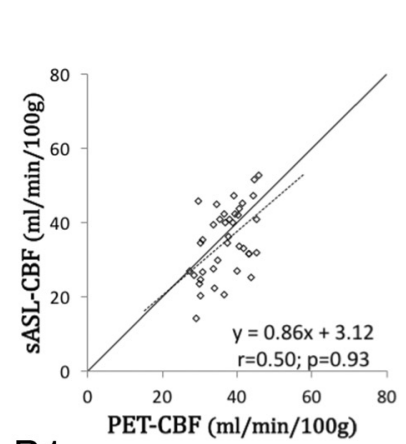

B1

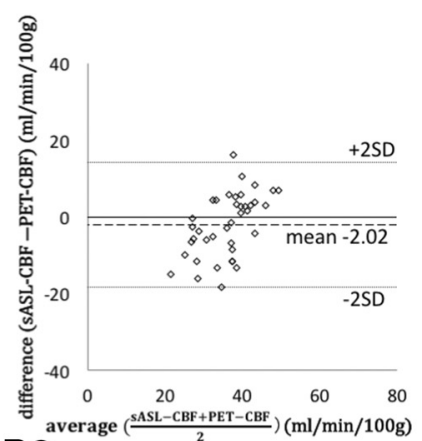

B2

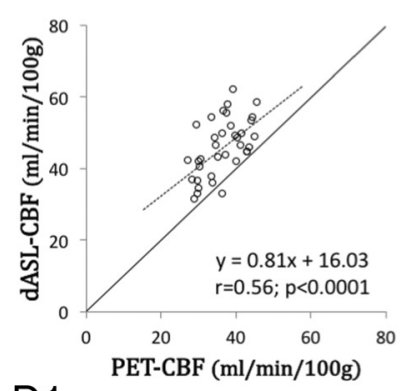

D1

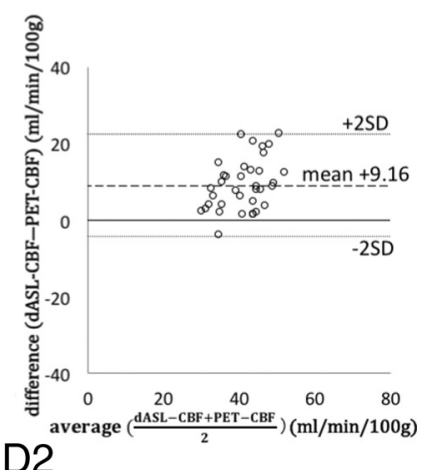

FIG 3. All plots between regional values of PET-CBF and those of $s A S L-C B F$ or $d A S L-C B F$ are divided by a Tmax cutoff of 6.0 seconds. A virtual straight line of PET-CBF equal to sASL-CBF is drawn in panels $A 1, B 1, C l$, and $D 1$. When the Tmax is $\leq 6.0$ seconds, the sASL-CBF values tend to overestimate the true $C B F(A 1, A 2)$, whereas they underestimate the true CBF when Tmax is $>6.0$ seconds $(B 1, B 2)$. On the other hand, $d A S L-C B F$ tends to overestimate the true CBF regardless of the Tmax, but the correlation of ASL and PET is better in areas with Tmax $>6.0$ seconds (Cl-C2, D1-D2).
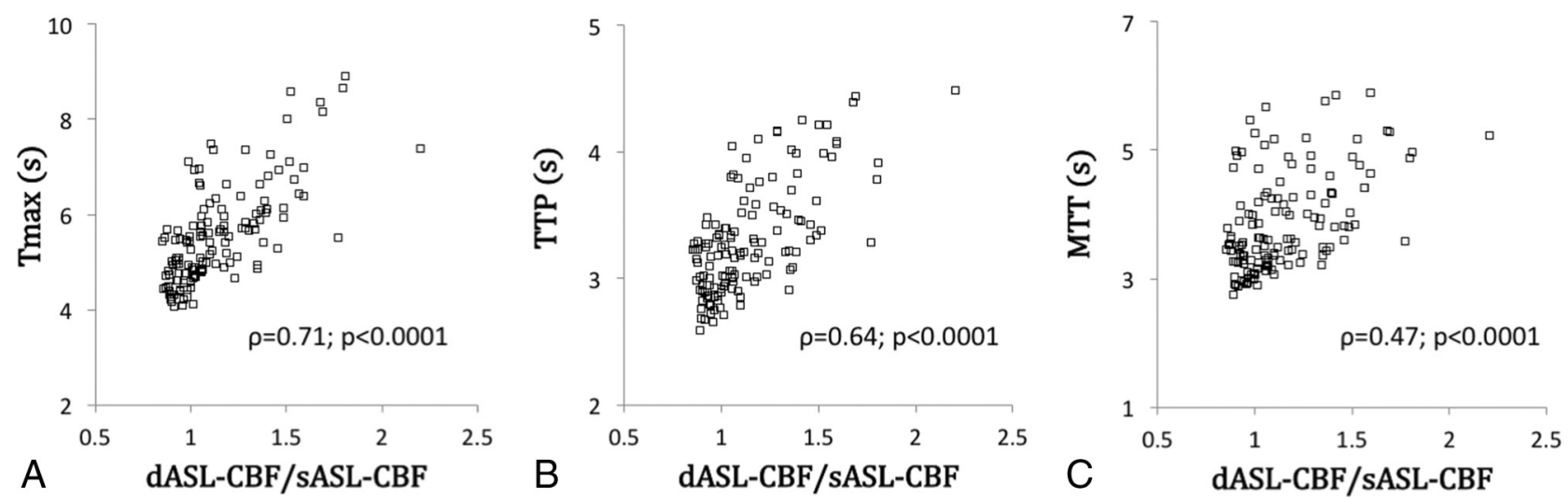

FIG 4. Correlations between the ratio of $d A S L-C B F$ to $S A S L-C B F$ and the DSC time-based parameters. Tmax, MTT, and TTP are well correlated with the ratio of $d A S L-C B F$ to $S A S L-C B F$.

Recently, Goetti et al ${ }^{20}$ reported a similar comparison study between ASL and PET in patients with Moyamoya disease, and they mentioned that cerebellar normalization improved the correlation between ASL and PET. We also tried the same method, but we found that the trend of overestimation was worse than that of the current method (On-line Fig 5). Our results showed that the ASL-CBF of the cerebellum was lower than that of average cortical regions, whereas PET-CBF was higher (ASL: average cortical regions, 64.4; cerebellum, 60.4; PET: average cortical regions, 59.9; cerebellum, 56.0). Several factors probably play a role in these different results, such as the difference in the patient populations, scanner settings, and measurement approaches for CBF by PET, and the difference in labeling efficiency between the anterior circulation and posterior circulation. However, we are unsure of the reasons for these different results because, to our knowledge, the normal values of cerebellar ASL-CBF have not been reported. Further data collection will be needed to clarify these differences.

Several groups have investigated the relationship between ASL-CBF and time-based parameters in patients with Moyamoya disease. Qiu et $\mathrm{al}^{26}$ assessed the relationship of CBF measured by pulsed continuous ASL with multiple PLDs and CBF values of Xe-CT. They reported that short PLDs (1.5-2 seconds) had the strongest correlation with CBF values obtained by Xe-CT ( $r=$ 
0.65), which overestimated the expected CBF in the short Tmax regions ( $\leq 4$ seconds) and underestimated Xe-CT CBF in the long Tmax areas ( $>4$ seconds). Conversely, the ASL of long PLDs $(>2.5$ seconds) was reported to underestimate Xe-CT CBF in the short Tmax regions, and the overestimation occurred in the long Tmax areas. Yun et $\mathrm{al}^{27}$ found that long TTP negatively affected the correlation of CBF values of ASL and DSC and that TTP delay ( $>10$ seconds) had a positive effect on the difference between ASL-CBF and DSC-CBF. In our study, the CBF values of sASL were much higher than those of PET-CBF in the less-affected regions, and the difference was small in the more-affected areas. On the other hand, the differences between dASL (ie, ASL of $\mathrm{PLD}=2525 \mathrm{~ms}$ ) and PET-CBF values were relatively constant in all groups regardless of perfusion delay. Based on the past studies, the principle of ASL, ${ }^{8-11}$ and our present results, most protons tagged by spin labeling would saturate brain tissue at the time of image acquisition with sASL in normally perfused areas, but they would not transit into the image acquisition area when the arterial transit time was long. Therefore, the sASL-CBF value dropped in the area with long transit time, and dASL-CBF was relatively constant. Taken together, these findings suggest that it would be difficult to obtain precise CBF values with the current ASL method in Moyamoya disease, even if using multiple PLDs. Longer PLD would be suitable for patients with longer transit time, but the problems of low SNR because of T1 decay would be aggravated. ${ }^{14}$

However, acquisition of ASL with multiple PLDs would nonetheless contribute to our understanding of the hemodynamics of Moyamoya disease. We observed an interesting method for turning the disadvantage of ASL (ie, the sensitivity of CBF values to perfusion delay) into an advantage by estimating the time-based parameters (Tmax, TTP, and MTT) based on the ratio of 2 ASLs (dASL-CBF to sASL-CBF). Several studies have explored the utility of time-based parameters calculated from ASL. Bokkers et $\mathrm{al}^{21}$ calculated the trailing-edge time of pulsed ASL at multiple delay times and revealed that the trailing edge was longer in patients with a carotid artery occlusion than in control patients and shorter in patients with leptomeningeal collaterals than in those without collaterals. Wolf et $\mathrm{al}^{22}$ used specific software to calculate the bolus arrival time of patients with acute ischemic stroke and found a significant correlation between ASL bolus arrival time and DSC-MTT $(r=0.28$ for all hemispheres, $r=0.42$ in the ischemic hemisphere). Our approach to the detection of perfusion delay yielded a better correlation than these studies. Compared with the respective PET-CBF estimations in areas with short time-based parameters, sASL underestimated PET-CBF and dASL-CBF overestimated PET-CBF in areas with long time-based parameters. These different effects induced by perfusion delay would result in the correlation of the ASL ratio and each of Tmax, TTP, and MTT.

Because time-based parameters are important to evaluating the degree of hemodynamic stress, noninvasive measurement of the perfusion delay by ASL would benefit children and adolescents with Moyamoya disease. Because MTT theoretically correlates with the reciprocal of tissue perfusion pressure, ${ }^{38}$ it should be a good indicator of profoundly decreased cerebral perfusion pressure in Moyamoya disease. ${ }^{4}$ In fact, an MTT delay cutoff of 2 seconds has been reported to detect misery perfusion ${ }^{5}$ and cutoff of 2.5 seconds has been reported to predict surgical revascularization $^{7}$ in patients with Moyamoya disease. Even if ASL is not suitable for the accurate measurement of CBF values in patients with Moyamoya disease, evaluation of the perfusion delay by ASL with multiple PLDs may be useful for evaluating the degree of hemodynamic stress and may contribute to decisions regarding patient treatment.

We analyzed only 18 cases with variable age and a mixture of clinical subtypes, and the subgroups we used for analysis contained smaller regions. Accordingly, some caution is necessary when interpreting the results. As discussed above, PLDs of 1525 ms and 2525 ms must be shorter than the arterial transit time of some patients, but we chose these PLDs in consideration of the acquisition time and SNR. The problem of low ASL values in the cerebellum compared with PET was still unresolved. Despite their preliminary nature, our data suggest that ASL is a promising clinical tool for evaluating patients with Moyamoya disease. Accumulation of more cases and comparison of data with healthy control patients and patients with atherosclerotic cerebrovascular disease will be needed to further explore the uses and efficacy of ASL.

\section{CONCLUSIONS}

Noninvasively measured ASL-CBF values had acceptable correlation with PET-measured absolute CBF, and a set of 2 ASLs using different PLDs can detect perfusion delay in patients with Moyamoya disease. Although we believe PET and DSC will continue to play important roles for investigating surgical indications and verifying clinical deterioration, ASL could be an alternative method for the initial screening of patients and for timed follow-up of both the operated and nonoperated cases, but only if we bear in mind its theoretic basis and limitations.

\section{ACKNOWLEDGMENTS}

The authors would like to thank Masako Akiyama for statistical guidance.

Disclosures: Yoji Tanaka_RELATED: Grants/Grants Pending: Japan Society for the Promotion of Science, Comments: KAKENHI 15K10294. Tadashi Nariai-RELATED: Grants/Grants Pending: SENSHIN Medical Research Foundation grant.

\section{REFERENCES}

1. Suzuki J, Takaku A. Cerebrovascular "Moyamoya” disease. Disease showing abnormal net-like vessels in base of brain. Arch Neurol 1969;20:288-99 CrossRef Medline

2. Matsushima Y. Moyamoya disease. In: Albright A, Pollack I, Adelson $\mathrm{P}$, eds. Principles and Practice of Pediatric Neurosurgery. Thieme: New York; 1999:1053-69

3. Research Committee on the Pathology and Treatment of Spontaneous Occlusion of the Circle of Willis, Health Labour Sciences Research Grant for Research on Measures for Intractable Diseases. Guidelines for diagnosis and treatment of Moyamoya disease (spontaneous occlusion of the circle of Willis). Neurol Med Chir (Tokyo) 2012;52:245-66 CrossRef Medline

4. Nariai T, Matsushima Y, Imae S, et al. Severe haemodynamic stress in selected subtypes of patients with Moyamoya disease: a positron emission tomography study. J Neurol Neurosurg Psychiatry 2005;76: 663-69 CrossRef Medline

5. Tanaka Y, Nariai T, Nagaoka T, et al. Quantitative evaluation of cerebral hemodynamics in patients with Moyamoya disease by dynamic susceptibility contrast magnetic resonance imaging-

AJNR Am J Neuroradiol 38:696-702 Apr 2017 www.ajnr.org 701 
comparison with positron emission tomography. JCereb Blood Flow Metab 2006;26:291-300 CrossRef Medline

6. Schubert GA, Weinmann C, Seiz M, et al. Cerebrovascular insufficiency as the criterion for revascularization procedures in selected patients: a correlation study of xenon contrast-enhanced CT and PWI. Neurosurg Rev 2009;32:29-35; discussion 35-36 CrossRef Medline

7. Ishii $Y$, Nariai T, Tanaka Y, et al. Practical clinical use of dynamic susceptibility contrast magnetic resonance imaging for the surgical treatment of Moyamoya disease. Neurosurgery 2014;74:302-09 CrossRef Medline

8. Williams DS, Detre JA, Leigh JS, et al. Magnetic resonance imaging of perfusion using spin inversion of arterial water. Proc Natl Acad Sci U S A 1992;89:212-16 CrossRef Medline

9. Alsop DC, Detre JA. Reduced transit-time sensitivity in noninvasive magnetic resonance imaging of human cerebral blood flow. J Cereb Blood Flow Metab 1996;16:1236-49 CrossRef Medline

10. Silva AC, Zhang W, Williams DS, et al. Estimation of water extraction fractions in rat brain using magnetic resonance measurement of perfusion with arterial spin labeling. Magn Reson Med 1997;37: 58-68 CrossRef Medline

11. Detre JA, Samuels OB, Alsop DC, et al. Noninvasive magnetic resonance imaging evaluation of cerebral blood flow with acetazolamide challenge in patients with cerebrovascular stenosis. J Magn Reson Imaging 1999;10:870-75 CrossRef Medline

12. Detre JA, Wang J, Wang Z, et al. Arterial spin-labeled perfusion MRI in basic and clinical neuroscience. Curr Opin Neurol 2009;22:348-55 CrossRef Medline

13. Hartkamp NS, van Osch MJ, Kappelle J, et al. Arterial spin labeling magnetic resonance perfusion imaging in cerebral ischemia. Curr Opin Neurol 2014;27:42-53 CrossRef Medline

14. Alsop DC, Detre JA, Golay X, et al. Recommended implementation of arterial spin-labeled perfusion MRI for clinical applications: a consensus of the ISMRM perfusion study group and the European consortium for ASL in dementia. Magn Reson Med 2015;73:102-16 CrossRef Medline

15. Ye FQ, Berman KF, Ellmore T, et al. H(2)(15)O PET validation of steady-state arterial spin tagging cerebral blood flow measurements in humans. Magn Reson Med 2000;44:450-56 CrossRef Medline

16. Kimura H, Kado H, Koshimoto Y, et al. Multislice continuous arterial spin-labeled perfusion MRI in patients with chronic occlusive cerebrovascular disease: a correlative study with $\mathrm{CO} 2$ PET validation. J Magn Reson Imaging 2005;22:189-98 CrossRef Medline

17. Bokkers RP, Bremmer JP, van Berckel BN, et al. Arterial spin labeling perfusion MRI at multiple delay times: a correlative study with $\mathrm{H}(2)(15) \mathrm{O}$ positron emission tomography in patients with symptomatic carotid artery occlusion. J Cereb Blood Flow Metab 2010;30: 222-29 CrossRef Medline

18. Xu G, Rowley HA, Wu G, et al. Reliability and precision of pseudocontinuous arterial spin labeling perfusion MRI on $3.0 \mathrm{~T}$ and comparison with 15O-water PET in elderly subjects at risk for Alzheimer's disease. NMR Biomed 2010;23:286-93 CrossRef Medline

19. Kilroy E, Apostolova L, Liu C, et al. Reliability of two-dimensional and three-dimensional pseudo-continuous arterial spin labeling perfusion MRI in elderly populations: comparison with 150-water positron emission tomography. J Magn Reson Imaging 2014;39: 931-39 CrossRef Medline

20. Goetti R, Warnock G, Kuhn FP, et al. Quantitative cerebral perfusion imaging in children and young adults with Moyamoya disease: comparison of arterial spin-labeling-MRI and $\mathrm{H}(2)[(15) \mathrm{O}]$-PET. AJNR Am J Neuroradiol 2014;35:1022-28 CrossRef Medline

21. Bokkers RP, van Laar PJ, van de Ven KC, et al. Arterial spin-labeling MR imaging measurements of timing parameters in patients with a carotid artery occlusion. AJNR Am J Neuroradiol 2008;29:1698-703 CrossRef Medline
22. Wolf ME, Layer V, Gregori J, et al. Assessment of perfusion deficits in ischemic stroke using 3D-GRASE arterial spin labeling magnetic resonance imaging with multiple inflow times. $J$ Neuroimaging 2014;24:453-924 CrossRef Medline

23. Lyu J, Ma N, Liebeskind DS, et al. Arterial spin labeling magnetic resonance imaging estimation of antegrade and collateral flow in unilateral middle cerebral artery stenosis. Stroke 2016;47:428-33 CrossRef Medline

24. Zaharchuk G, Do HM, Marks MP, et al. Arterial spin-labeling MRI can identify the presence and intensity of collateral perfusion in patients with Moyamoya disease. Stroke 2011;42:2485-91 CrossRef Medline

25. Saida T, Masumoto T, Nakai Y, et al. Moyamoya disease: evaluation of postoperative revascularization using multiphase selective arterial spin labeling MRI. J Comput Assist Tomogr 2012;36:143-49 CrossRef Medline

26. Qiu D, Straka M, Zun Z, et al. CBF measurements using multidelay pseudocontinuous and velocity-selective arterial spin labeling in patients with long arterial transit delays: comparison with xenon CT CBF. J Magn Reson Imaging 2012;36:110-19 CrossRef Medline

27. Yun TJ, Sohn $\mathrm{CH}$, Han $\mathrm{MH}$, et al. Effect of delayed transit time on arterial spin labeling: correlation with dynamic susceptibility contrast perfusion magnetic resonance in Moyamoya disease. Invest Radiol 2013;48:795-802 CrossRef Medline

28. Noguchi T, Kawashima M, Irie H, et al. Arterial spin-labeling MR imaging in Moyamoya disease compared with SPECT imaging. Eur J Radiol 2011;80:e557-62 CrossRef Medline

29. Noguchi T, Kawashima M, Nishihara M, et al. Arterial spin-labeling MR imaging in Moyamoya disease compared with clinical assessments and other MR imaging findings. Eur J Radiol 2013;82: e840-47 CrossRef Medline

30. Donahue MJ, Ayad M, Moore R, et al. Relationships between hypercarbic reactivity, cerebral blood flow, and arterial circulation times in patients with Moyamoya disease. J Magn Reson Imaging 2013;38: 1129-39 CrossRef Medline

31. Wang R, Yu S, Alger JR, et al. Multi-delay arterial spin labeling perfusion MRI in Moyamoya disease-comparison with CT perfusion imaging. Eur Radiol 2014;24:1135-44 CrossRef Medline

32. Houkin K, Nakayama N, Kuroda S, et al. Novel magnetic resonance angiography stage grading for Moyamoya disease. Cerebrovasc Dis 2005;20:347-54 CrossRef Medline

33. Ostergaard L, Weisskoff RM, Chesler DA, et al. High resolution measurement of cerebral blood flow using intravascular tracer bolus passages. Part I: mathematical approach and statistical analysis. Magn Reson Med 1996;36:715-25 CrossRef Medline

34. Ostergaard L, Weisskoff RM, Chesler DA, et al. High resolution measurement of cerebral blood flow using intravascular tracer bolus passages. Part II: experimental comparison and preliminary results. Magn Reson Med 1996;36:726-36 CrossRef Medline

35. Senda M, Buxton RB, Alpert NM, et al. The 150 steady-state method: correction for variation in arterial concentration. J Cereb Blood Flow Metab 1988;8:681-90 CrossRef Medline

36. Sadato N, Yonekura $Y$, Senda M, et al. PET and the autoradiographic method with continuous inhalation of oxygen-15-gas: theoretical analysis and comparison with conventional steady-state methods. J Nucl Med 1993;34:1672-80 Medline

37. Iida H, Kanno I, Miura S, et al. Error analysis of a quantitative cerebral blood flow measurement using $\mathrm{H} 2$ (15)O autoradiography and positron emission tomography, with respect to the dispersion of the input function. J Cereb Blood Flow Metab 1986;6:536-45 CrossRef Medline

38. Powers WJ, Grubb RL Jr, Raichle ME. Physiological responses to focal cerebral ischemia in humans. Ann Neurol 1984;16:546-52 CrossRef Medline 